\title{
WOX family genes in somatic embryogenesis
}

\author{
Krasnoperova E.Y.*, Tvorogova V.E., Lutova L.A. \\ Saint-Petersburg State University, St. Petersburg, Russia \\ *email: eliz.krasnoperova@gmail.com
}

Somatic embryogenesis is one of the plant regeneration ways. In this process embryos are formed not from generative, but from vegetative tissues. It is widely used in biotechnology for plant transformation. The genes, participating in this process, usually also function in the zygotic embryogenesis. The homeodomain-containing transcription factors of the WOX family are important regulators of plant development, including both the somatic and zygotic embryogenesis. In our study the participation of the Medicago truncatula MtWOX9-1 gene in somatic embryogenesis was shown. Its overexpression leads to a significant increase of somatic embryogenesis capacity of callus tissue and to changes in expression levels of a number of genes, involved in somatic embryogenesis. The aim of this study is to analyse the interaction of MtWOX9-1 products with other genes, associated with somatic embryogenesis. Also, transcription factors from WOX family were shown to regulate expression of CLE family genes. We supposed that $M t C L E 6$ and $M t C L E 18$ genes can also be directly regulated by MtWOX9-1 transcription factor, because, according to the results of transcriptomic analysis, overexpression of MtWOX9-1 changes the expression of these genes. We use the ChIP-Seq and EMSA method to check this hypothesis.

Along with WOX9, WOX2 in A. thaliana is known to regulate zygotic embryogenesis. We found an elevation in the level of expression of its ortholog, MtWOX2, during somatic embryogenesis. Moreover, some calli with MtWOX2 overexpression are characterised by increased weight and due to this, increased amount of somatic embryos. Now we are carrying out a transcriptome analysis of calli with overexpression of this gene. Acknowledgements: The research was supported by the RFBR (20-016-00124). 